\title{
La vocation de Baie-Saint-Paul et de sa région ${ }^{1}$
}

\author{
Marc-Urbain Proulx \\ Université du Québec à Chicoutimi
}

\section{Introduction}

Ce qui frappe à prime abord le spécialiste en développement local et régional lorsqu'il se penche sur le cas de Baie-Saint-Paul et de sa région, c'est la qualité des études dont ce territoire fut doté au cours des dernières décennies. Baie-Saint-Paul et les autres entités municipales, les deux municipalités régionales de comté (MRC) et la grande région historique de Charlevoix bénéficient en effet de plusieurs documents de réflexion pour visualiser, comprendre et analyser les composantes écologiques, historiques, sociales, culturelles, politiques et économiques de son territoire. Ce qui frappe ensuite l'analyste, ce sont les paradoxes vécus sur ce territoire en quête de développement et d'équilibre. En voici quelques-uns :

- Charlevoix détient, certes, le titre de « région naturelle » dans le sens géographique. Or, cette région est non seulement coupée en deux MRC distinctes, mais elle doit aussi subir une attache politique et administrative à la région de la capitale nationale;

- cette attache à une région métropolitaine soulève un autre paradoxe puisque Charlevoix possède non seulement un caractère rural, mais s'avère, sous l'angle économique, une région-ressources;

- le tourisme et la villégiature apportent des retombées économiques considérables aux habitants de Baie-Saint-Paul qui, en retour, paient malheureusement une importante taxe saisonnière dissimulée sous la triple forme d'une flambée des prix, d'une limite d'accès « au pays » et d'une perte de qualité de vie;

- le paysage offert par ce territoire représente sa condition première de prospérité grâce à la demande de villégiature; consommation de villégiature qui cause cependant en Charlevoix des effets négatifs de plus en plus compromettants pour l'attractivité de ce paysage;

- la vocation «ville d'art et de culture » de BaieSaint-Paul bénéficie d'une reconnaissance mondiale de plus en plus confirmée, mais elle peine néanmoins à être pleinement reconnue à l'échelle locale comme une image de marque et de marketing;

- au contraire des autres régions du Québec, Charlevoix n'a pas bénéficié d'un véritable décollage industriel au cours de son histoire économique, mais ce territoire préconise encore aujourd'hui une politique industrielle pour soutenir son développement;

- Baie-Saint-Paul et sa périphérie misent actuellement sur l'émergence d'un nouveau savoir-faire agroalimentaire, alors que son inestimable savoirfaire agricole patrimonial s'effrite très rapidement depuis trois décennies;

- alors que les jeunes scolarisés sont en exode vers d'autres cieux, on recrute péniblement et difficilement de la main-d'œuvre spécialisée et des savoirfaire dans les secteurs économiques traditionnels et émergents.

Ce ne sont là que quelques paradoxes qui illustrent les contradictions inhérentes au développement local et régional tel que conçu habituellement. Nous les illustrons en introduction de notre allocution tout simplement pour postuler que Baie-Saint-Paul (et Charlevoix) recherchent plus ou moins explicitement un nouvel équilibre sous plusieurs dimensions. Ces dimensions sont bien éclairées dans le document de 
réflexion conçu spécialement pour ce Sommet économique. Document qui permettra par ailleurs aux lecteurs de soulever spécifiquement à leur mémoire leurs besoins, leurs préférences et leur volonté.

Pour notre contribution au débat et au processus collectif, nous ferons, dans ce texte, une lecture historique et contemporaine des stratégies de développement appliquées dans cette région de Charlevoix dont Baie-Saint-Paul représente un pôle principal. L'exercice débouchera sur le traitement de la stratégie territoriale très actuelle du gouvernement du Québec à l'égard des " créneaux territoriaux ». Baie-Saint-Paul doit faire des choix et se positionner d'une manière stratégique. Nous proposerons alors la mise en œuvre d'un nouvel outil de développement territorial s'appuyant sur les Technologies de l'information et de la communication (TIC) pour relever quatre enjeux stratégiques afin que le milieu soit davantage innovateur, producteur et créateur de richesses.

\section{L'octroi de bassins de ressources}

L'offre de bassins de ressources naturelles représente la stratégie classique utilisée par les autorités publiques afin de diffuser le développement dans les périphéries. À Baie-Saint-Paul, Pierre Dupré reçut, en 1672 , la première concession de la seigneurie du Gouffre. On exploita alors les terres et la forêt. À partir de 1716, les colonisateurs arrivèrent, attirés par les belles terres offertes dans les vallées intérieures, sur les plateaux et sur les rivages. Il y avait aussi de riches forêts. D'autres colons sont ainsi venus en Charlevoix à partir de portes d'entrée telles que La Malbaie, Saint-Siméon, les Éboulements et autres, avec la même motivation. Aussi, les ressources maritimes ont attiré des pêcheurs, des marins (pilotes) et ensuite des villégiateurs. Les revenus générés par toutes ces activités reliées aux ressources naturelles ont permis à Charlevoix de sortir d'une économie traditionnelle d'auto-subsistance et d'entrer doucement dans la modernité en utilisant parcimonieusement les surplus financiers pour doter les divers lieux en équipements et en services collectifs dans l'éducation, la santé, la voirie, la culture, etc.

Mis à part la ville de Clermont, la région de Charlevoix n'a jamais connu de véritable décollage industriel vigoureux à la manière du Saguenay-Lac-SaintJean, de la Côte-Nord, de la Mauricie et de l'Abitibi-

Témiscamingue. Coincée entre les montagnes, la mer et la rivière Saguenay, Charlevoix ne contient pas une très grande réserve de ressources naturelles, finalement. L'exploitation forestière s'avère limitée. Il n'y a pas beaucoup de ressources minérales. Le nombre d'excellentes terres à cultiver est peu élevé. La rupture des stocks de mines et de pêche, la saturation des terres ainsi que les limites de la forêt obligèrent même assez rapidement les jeunes Charlevoisiens à l'émigration vers le Saguenay limitrophe ouvert à la colonisation en 1838 , vers les vastes chantiers nord côtiers d'une certaine époque et aussi vers les grands centres urbains.

En cette matière de ressources naturelles, l'offre d'aujourd'hui réside principalement dans le magnifique environnement dont est doté Charlevoix, notamment le Massif de la petite rivière Saint-François, l'île aux Coudres, le Massif des Grands Jardins, les Hautes Gorges, le fjord du Saguenay, le littoral du fleuve, les caps, les baies, les vals, les pics, les lacs. Il existe aussi plusieurs bonnes terres agricoles plus ou moins en friche. Bref, Baie-Saint-Paul et Charlevoix sont encore un " pays » attractif, de villégiateurs bien sûr, mais aussi d'entreprises.



L’aménagement du territoire

Ici comme ailleurs, l'aménagement du territoire fut d'abord l'œuvre des colons et des investisseurs qui construisirent des habitations, des bâtiments, des fermes, des moulins à scie et à farine, des chapelles, des églises et des couvents, des auberges et des résidences ainsi que des hôtels. Par la suite, les gouvernements locaux et centraux se préoccupèrent de leur mission d'aménagement qui devint, en réalité, une véritable stratégie de développement territorial. Ponts, chemins, routes, écoles, ports ont ainsi permis de donner une forme explicite à l'espace occupé, tout en permettant une meilleure exploitation des ressources.

Enclavée entre les Laurentides, le Saguenay et le fleuve, Charlevoix fut rapidement désenclavé grâce à 
ses quais et à ses ports. Par la suite, on aménagea une route vers Québec en 1825 puis une voie ferrée qui permit l'arrivée du premier train à Baie-Saint-Paul en 1914. Sillonnant le fleuve jusqu'à Malbaie (Clermont) en charriant marchandises, voyageurs et espoirs de prospérité, cette voie ferrée fut longtemps l'épine dorsale du développement économique. Ce développement s'effectua en conservant néanmoins plusieurs éléments de traditions.

Tout le $\mathrm{Xx}^{\mathrm{e}}$ siècle fut une époque d'aménagement extensif du vaste territoire québécois par le secteur public, notamment après 1945 avec les réseaux de collèges, d'aqueduc et d'égouts municipaux, de routes bitumées, d'hôpitaux, de polyvalentes, de CLSC, de centres communautaires, etc. On a aussi construit des équipements structurants plus sophistiqués tels que des hôpitaux spécialisés, des collèges, des universités et des centres spécialisés. La figure 1 nous permet de constater que Baie-Saint-Paul et Charlevoix ne furent pas en reste, même si chaque dossier a nécessité beaucoup de démarchage. La ville obtint notamment un centre hospitalier spécialisé qui s'est avéré un important moteur de développement économique. Et que dire du rôle actuel du centre d'art et du centre d'exposition? En réalité, Baie-Saint-Paul et Charlevoix possèdent plusieurs équipements (mineurs et majeurs) qui consolident leur créneau nature-culture.

Figure 1 - Principaux équipements publics aménagés en Charlevoix

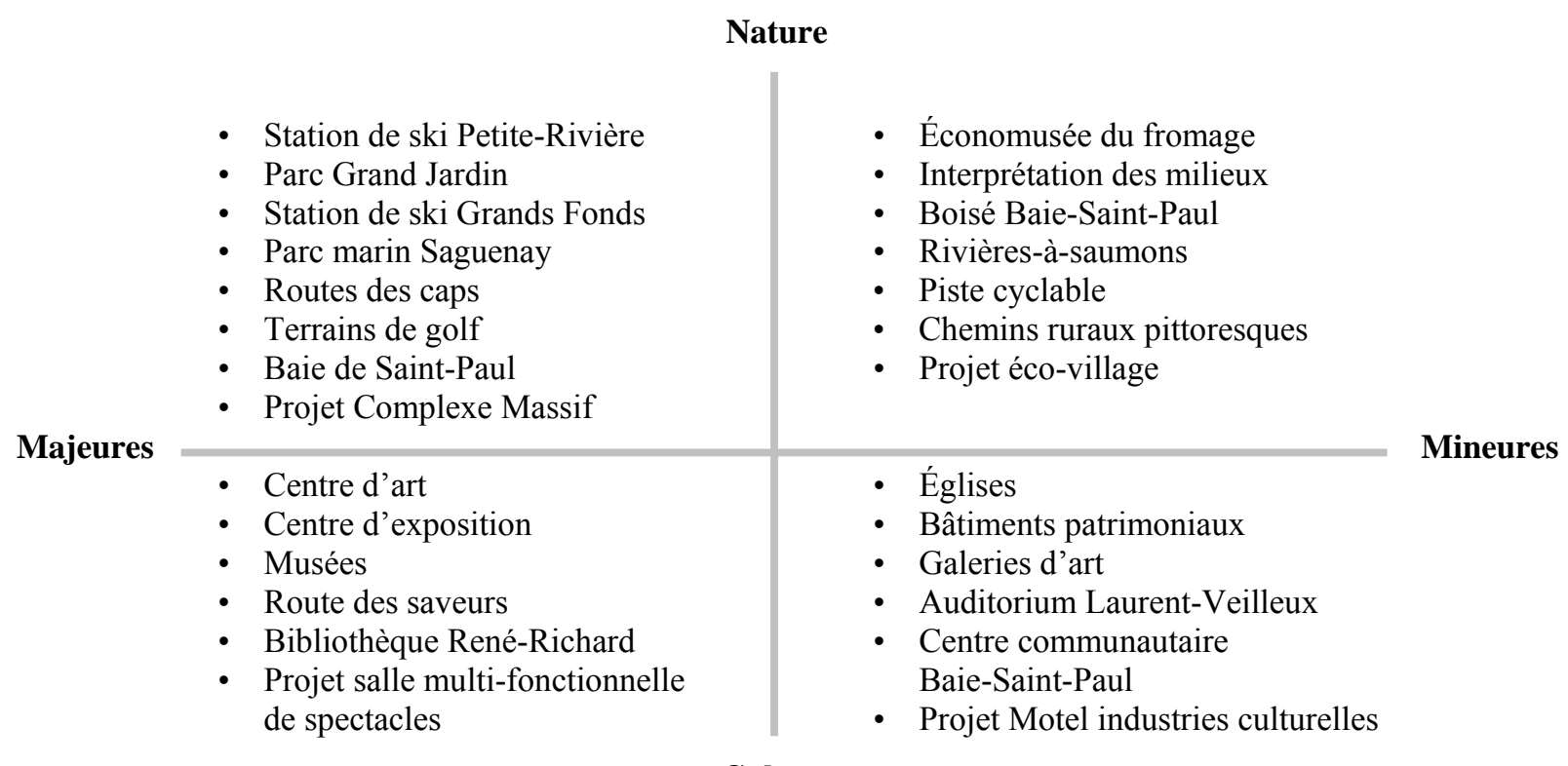

Culture

\section{Stratégie de polarisation}

Étant donné la dispersion des ressources naturelles et de la population, l'aménagement du territoire a favorisé la multiplication de petits pôles d'équipements et de services (figure 1) à travers l'espace. Le Charlevoix traditionnel est réellement un territoire polycentrique ou multipolaire, dont certains centres se démarquent davantage par leur capacité d'attirer des équipements, des entreprises, des services publics et la population.

À la fin des années 1960, Baie-Saint-Paul fut désigné comme l'un des deux principaux pôles de développement dans sa région. La proximité de Québec, qui concentre de nombreux services spécialisés, et la dimension limitée de sa périphérie ont cependant fortement contraint la polarisation et le rayonnement de ce centre urbain. Néanmoins, à partir de ses institutions religieuses, de ses commerces, de son centre hospitalier spécialisé, de ses écoles, Baie-Saint-Paul a bénéficié, à titre de pôle régional, de plusieurs équipements structurants tels que la polyvalente, le centre d'art, le centre d'exposition, la bibliothèque et d'autres services supérieurs. Dans sa région multipolaire, le pôle Baie-Saint-Paul trouve à l'évidence sa vocation première dans les services supérieurs autant privés que publics qui forment en réalité cinq foyers principaux de polarisation d'activités économiques (figure 2). 


\section{Figure 2 - Les cinq foyers de polarisation de Baie-Saint-Paul}

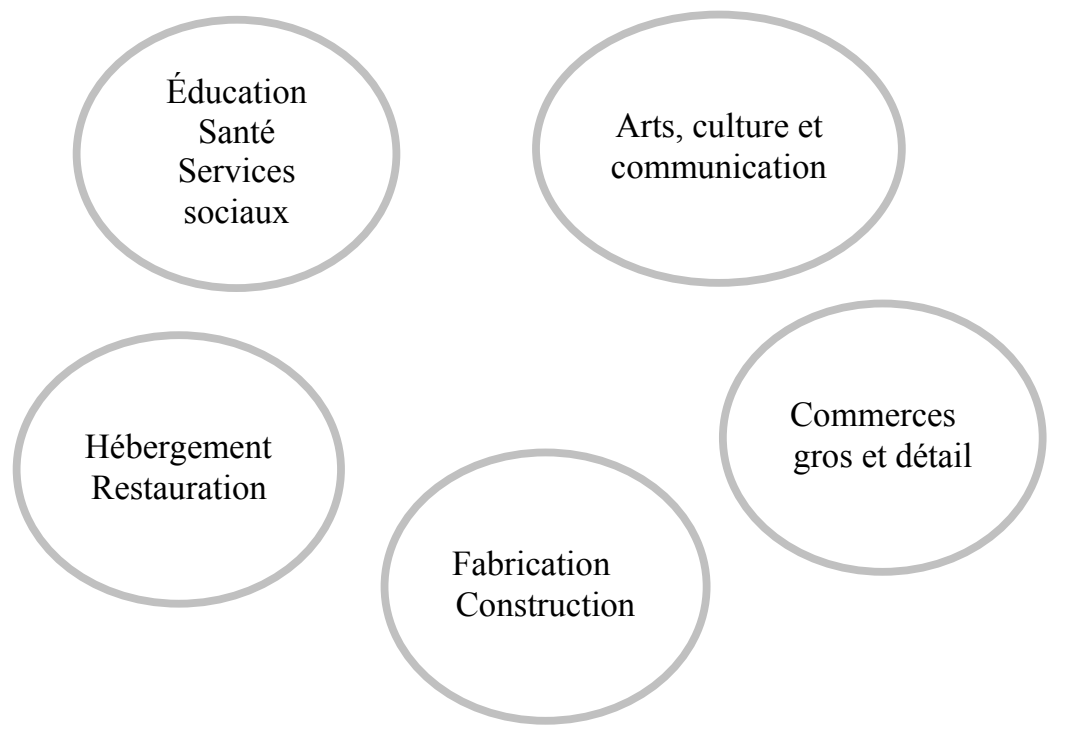

Puisque le centre-ville de ce pôle est déjà congestionné et que le précieux patrimoine bâti en ce lieu subit de nombreuses pressions, la déconcentration des activités représente certes une solution optimale. Nous recommandons alors à Baie-Saint-Paul l'adoption d'une solide politique de déconcentration s'appuyant sur deux couronnes territoriales :

- déconcentration périurbaine des activités industrielles (y compris les industries culturelles), des grandes surfaces commerciales, de l'hébergement de masse et de la restauration rapide;

- déconcentration périphérique des activités de plein air, d'interprétation de l'environnement, de contemplation des paysages, de loisirs spécialisés et d'un éventuel parc d'amusement.

La région polycentrique de Charlevoix doit confirmer son aménagement ordonné en plusieurs pôles d'activités selon un esprit de complémentarité plutôt que de concurrence. Elle doit limiter la forte tendance à la concentration des activités. Les règlements d'urbanisme et les schémas d'aménagement des MRC devraient être confectionnés en conséquence, avec des zonages restrictifs à vocation spécifique.

\section{Stratégie de construction régionale}

Charlevoix n'a pas obtenu le statut officiel de région lors du découpage du Québec en régions adminis- tratives à la fin des années 1960. Elle fut donc très peu dotée d'organisations publiques déconcentrées telles que les directions régionales des ministères et les conseils régionaux. Lors de la création des MRC au tournant des années 1980, le sentiment identitaire dual de la population et des élites a favorisé l'établissement de deux territoires supralocaux distincts. La région naturelle de Charlevoix demeure sans statut formel. Pourtant, il apparaît tout à fait logique de considérer la nécessité d'une vision charlevoisienne du développement en favorisant celui-ci par la mise en place d'équipements, de services, d'actions et d'initiatives d'envergure régionale.

Or, la construction du grand Charlevoix est bel et bien en cours. D'abord, parce que la population considère implicitement son inéluctabilité puisque Charlevoix est réellement une région naturelle comme pas une. Ensuite, parce qu'il existe une vision globale de plus en plus explicite, se référant généralement à la dénomination historique et prestigieuse de Charlevoix. Aussi, il est important de souligner la présence de plusieurs institutions communes telles que l'Association touristique, la commission scolaire, la radio communautaire, les deux journaux hebdomadaires, la table agrotouristique, la Commission de formation professionnelle. Finalement, existent en Charlevoix des outils communs d'aménagement et de développement comme la Route des saveurs, la Route du fleuve, le projet de pistes cyclable, la Randonnée nature Charlevoix, etc., sans oublier le concept de « réserve de la biosphère » (figure 1). 
La construction régionale de Charlevoix possède son originalité et sa propre force créatrice dont le déploiement ne fait que commencer. Alors que la majorité des régions administratives du Québec sont institutionnellement cristallisées à degrés divers, Charlevoix possède, à cet égard de dynamique institutionnelle, un potentiel considérable qui peut s'appuyer sur un grand nombre de ressources et d'équipements communs à aménager, à gérer et à intégrer selon une vision globale du développement de ce territoire. $\mathrm{Ne}$ serait-ce qu'un service de R\&D en aubergerie et dans les produits du terroir, qui apparaît actuellement si nécessaire. D'autres outils de développement émergeront à l'évidence de la dynamique organisationnelle de la grande région, notamment dans ce créneau nature-culture.

\section{Stratégie d'interaction}

Charlevoix possède peu d'institutions communes à l'échelle de la grande région pourtant traversée par des flux importants (marchandises, travailleurs, touristes, fonctionnaires, consommateurs....) qui tissent des relations d'affaires privées et publiques. Charlevoix n'a en réalité que peu profité de la stratégie régionale de développement de Québec et d'Ottawa basée sur la mise en place de structures de concertation entre les acteurs, tout en agissant tout de même considérablement en ce sens.

\section{Alors que la majorité des régions administratives du Québec sont institutionnellement cristallisées à degrés divers, Charlevoix possède, à cet égard de dynamique institutionnelle, un potentiel considérable.}

Cette stratégie d'interaction trouve ses origines dans les activités traditionnelles des chambres de commerce et des clubs spécialisés. Elle fut systématiquement mise en œuvre au Québec, dans les années 1970, avec les conseils régionaux (culture, environnement, santé et services sociaux, développement, etc.) dont le rôle de concertation était explicite. Au début des années 1980, la création de comités, corporations, associations et sociétés de développement fut soutenue à l'échelle des MRC, notamment les sociétés d'aide au développement des collectivités (SADC) et plus tard les centres locaux de développement (CLD). On a vu aussi se multiplier les tables, comités et commissions sectorielles ou thématiques de concertation ainsi que les forums, conférences socio-économiques et sommets sur les territoires MRC plus dynamiques. Tous ces mécanismes ou formules d'interaction visaient essentiellement à animer et à soutenir, par l'entremise de la concertation, le processus collectif d'apprentissage au développement territorial.

Un tel processus d'apprentissage collectif au développement de la grande région de Charlevoix existait bel et bien, mais d'une manière aléatoire. Nous pouvons identifier concrètement trois types de mécanismes institutionnels d'interaction regroupés dans la figure 3. Ces mécanismes d'interaction doivent, à notre avis, être systématiquement renforcés afin de soutenir encore davantage l'animation du développement régional à l'échelle du grand Charlevoix. Le Sommet économique de Baie-Saint-Paul représente un bel exemple à poursuivre. Nous recommandons ainsi aux autorités de Baie-Saint-Paul de jouer un véritable rôle de promoteur de cet apprentissage collectif au développement régional de Charlevoix en multipliant les occasions de concertation élargie sur des dossiers concrets.

\section{La stratégie des créneaux territoriaux}

À l'instar de la plupart des pays de l'OCDE (Organisation de coopération et de développement économique), le Québec est à l'heure de la diversification des économies territoriales à l'intérieur de leurs spécialités propres, en multipliant les opérations de transformation et de valeur ajoutée. Le concept de filière de production ou de cluster, appelés aussi grappes, sert d'ancrage théorique à cette stratégie de développement territorial s'appuyant sur les créneaux territoriaux à fort potentiel.

On a ainsi vu apparaître, au cours des années 1990, les Cités du multimédia, de l'électronique et de l'optiquephotonique, les technopoles maritime, de l'agroalimentaire et de l'aérospatial ainsi que la Vallée de l'aluminium. Depuis 2002, le programme ACCORD de la Société générale de financement cherche à généraliser cette stratégie de créneaux en désignant quelques créneaux dans chacune des régions administratives, notamment l'éolien en Gaspésie, la phytogénétique sur la Côte-Nord, la tourbe dans le Bas-SaintLaurent, le récréotourisme dans les Laurentides, les 
technomines souterraines en Abitibi. On voit aussi apparaître une variété de petits créneaux ou niches dans l'agroalimentaire comme l'agneau dans le Basdu-fleuve, le bioalimentaire ici et là, les bleuets au
Lac-Saint-Jean et le veau dans Charlevoix. Ces productions de niches sont aussi soutenues par la politique de la ruralité.

Figure 3 - Mécanismes institutionnels d'interaction en Charlevoix

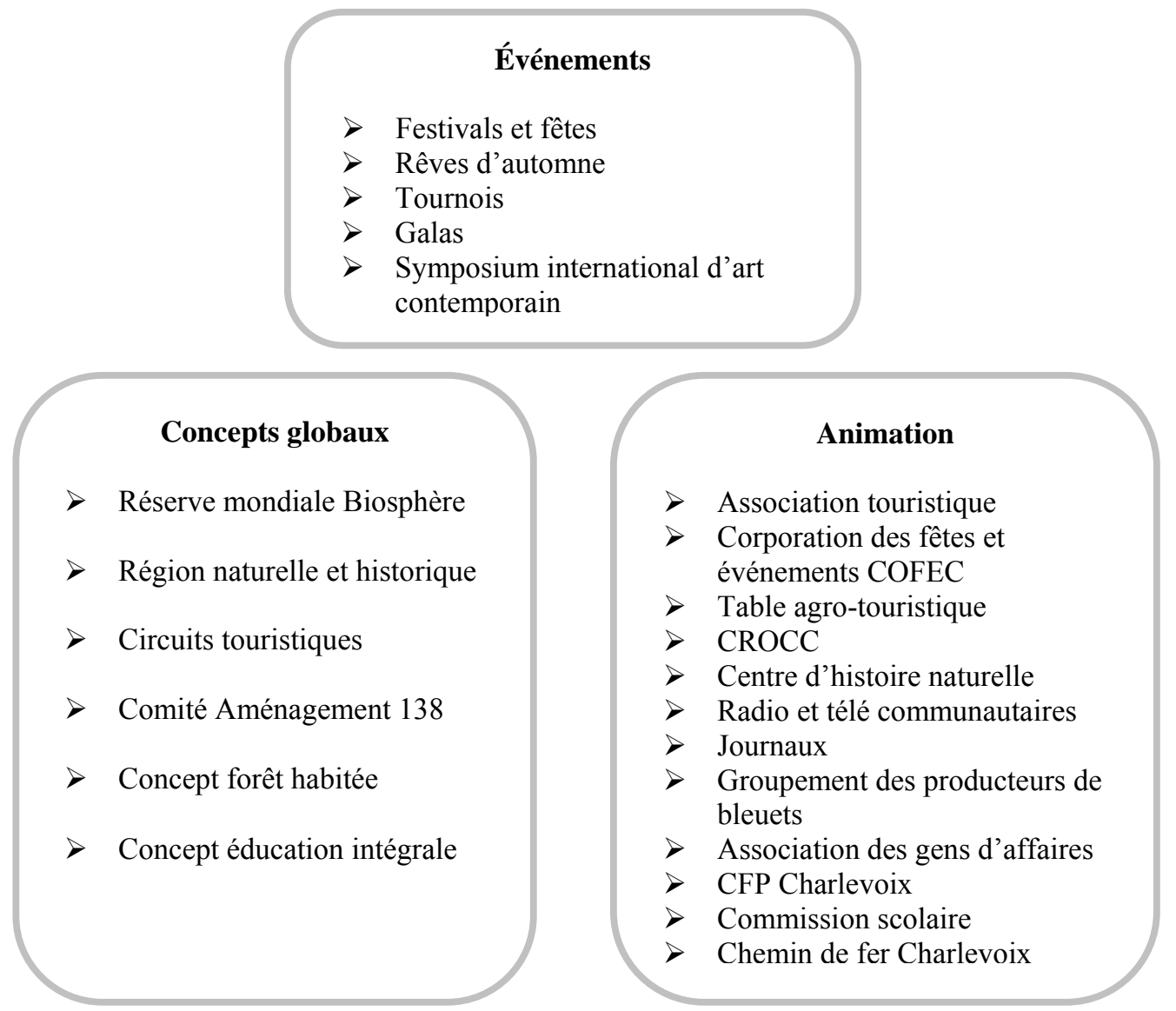

La nouvelle stratégie de développement basée sur les créneaux territoriaux vise en premier lieu à favoriser les investissements privés, stimulés notamment par la Société générale de financement (SGF) et Investissements Québec. Selon les forces et les faiblesses de la filière de production visualisée tel un champ concurrentiel en évolution, les mesures du secteur public peuvent aussi être orientées dans un esprit de structuration du créneau. Nous pensons notamment aux mesures actuelles à l'égard de la formation professionnelle, de la planification des affaires, du financement du risque et de l'animation économique en général qui peuvent éventuellement être reciblées.

À Baie-Saint-Paul et en Charlevoix, le couple natureculture (figure 1) apparaît tel un créneau territorial possédant un important potentiel de structuration et de développement. Notre analyse des acteurs endogè- nes et exogènes concernés par un tel créneau, à la lumière de la théorie du développement et des acquis actuels, nous permet d'identifier quatre grands enjeux stratégiques à relever par le milieu, qui sont aussi illustrés dans la figure 4 :

- l'enjeu de la veille informationnelle (marchés, technologie, concurrence, réglementations, etc.) concerne la nécessité de fournir collectivement aux entreprises de Baie-Saint-Paul et de la région de l'information pertinente à l'innovation et à la production;

- l'enjeu du marketing territorial fait référence à la visibilité et au positionnement du créneau natureculture de Baie-Saint-Paul sur le vaste marché afin de vendre ses produits, bien sûr, mais aussi de prospecter des entreprises, des savoir-faire et des 
capitaux qui correspondent à des segments faibles de la filière actuelle de production de biens et services;

- l'enjeu de la réponse aux clients concerne l'adaptation et la modulation optimales de la desserte de biens et services publics (éducation, santé, loisirs, formation....) aux véritables besoins des citoyens, des travailleurs et des entreprises;

- finalement, l'enjeu de la coordination des acteurs relève de la nécessité de créer le maximum de cohérence entre les diverses actions publiques et collectives de soutien territorial (financement, équipements, $\mathrm{R} \& \mathrm{D}$, animation, etc.) au développement socio-économique.

Afin de relever adéquatement ces enjeux stratégiques reliés au créneau nature-culture en Charlevoix, nous proposons aux acteurs de Baie-Saint-Paul la mise en œuvre d'un outil d'appropriation collective des TIC (Technologies d'information et de communication). Un certain nombre de conditions initiales doivent alors être envisagées, notamment un leadership fort de la ville de Baie-Saint-Paul comme maître d'œuvre de ce nouvel outil collectif.

Figure 4 - Enjeux stratégiques du créneau nature-culture

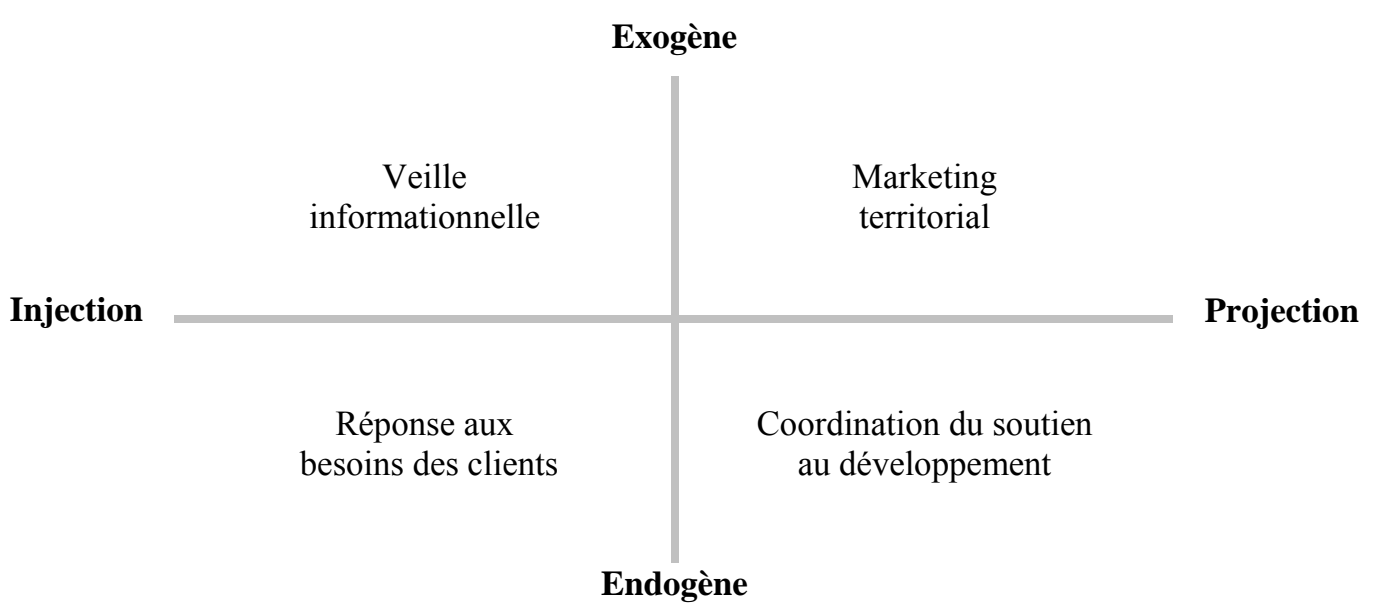

\section{Conclusion}

Le destin de Baie-Saint-Paul est intimement lié à celui de sa grande région naturelle et historique. Comme tout territoire qui recherche l'équilibre dans le développement, Baie-Saint-Paul et Charlevoix vivent des paradoxes et des contradictions, notamment entre les conditions du bon fonctionnement de l'économie et celles de la préservation de la qualité de vie.

Baie-Saint-Paul et Charlevoix offrent aux acteurs du développement un magnifique environnement qui contient plusieurs composantes majeures telles que deux massifs, des forêts, l'île aux Coudres, le littoral du fleuve, les Hautes Gorges, les plateaux, les vallées, les caps, les rivières, etc. Malgré la grande richesse visuelle et l'apparente abondance, les ressources naturelles de cet environnement ne furent pas suffisantes dans le passé pour occasionner un véritable décollage industriel ni même pour générer beaucoup de richesses. Ainsi, les habitants furent obligés à l'émigration vers d'autres cieux. Ces ressources demeurent aujourd'hui très fragiles.

Par ailleurs, la stratégie d'aménagement du territoire des gouvernements supérieurs a considérablement bénéficié à Baie-Saint-Paul et à Charlevoix en les dotant d'infrastructures et d'équipements publics, notamment dans le transport, la santé et les arts. Cet aménagement de Charlevoix a non seulement désenclavé cette région, mais a aussi confirmé son caractère polycentrique ou multipolaire. L'enjeu principal d'aujourd'hui dans ce domaine stratégique concerne les équipements à l'échelle de la grande région naturelle, en particulier un centre de formation et de $R \& D$ en aubergerie et en produits du terroir. Des institutions régionales sont aussi à mettre en œuvre sur la base des acquis actuels. 
Nous avons constaté que Baie-Saint-Paul possède cinq foyers de polarisation des activités et de la population. Cependant, la congestion, l'encombrement et la baisse de la qualité de vie des citoyens au centre de cette ville militent actuellement en faveur d'une politique municipale articulée à l'égard de la déconcentration des activités dans la couronne périurbaine et dans la périphérie immédiate et éloignée, sans cependant affecter la vitalité économique des activités centrales.

Le Québec est actuellement à l'heure de la diversification économique sur la base des créneaux territoriaux. Le couplage entre nature et culture représente un créneau à très fort potentiel pour Baie-Saint-Paul et Charlevoix. De nombreux équipements, services et actions collectives s'inscrivent déjà en ce sens. Afin de structurer cette filière ou ce créneau, nous avons proposé aux acteurs du développement de Baie-Saint-
Paul un outil collectif capable de favoriser l'appropriation des TIC. Dans le cadre du créneau en question, cette appropriation des TIC doit s'effectuer autour de quatre enjeux stratégiques à relever par la collectivité, soit la veille informationnelle, le marketing territorial, la réponse aux besoins des clients et la coordination des acteurs dans un esprit de cohérence globale du soutien au développement économique, social et culturel.

\section{Nous avons constaté que Baie-Saint-Paul possède cinq foyers de polarisation des activités et de la population.}

\section{Note}

Ce texte fut livré à l'ouverture du Sommet économique de Baie-Saint-Paul, le 25 avril 2003. 